\title{
Atypical Presentation of Sacrococcygeal Pilonidal Disease: Presentation and Surgical Management
}

\author{
Ahmed Al Wadiya ${ }^{1 *}$, Amr Abouelela ${ }^{1}$, Abdallah Al Ghunimat ${ }^{2}$, Neil \\ Ashwood $^{3}$, Amit Kotecha ${ }^{3}$ and Ahmad Gharaibeh ${ }^{4}$ \\ ${ }^{1}$ Trauma \& Orthopaedics surgeon-Specialist, University hospitals of Derby and Burton, UK \\ ${ }^{2}$ Trauma \& Orthopaedics Junior surgeon, University hospitals of Derby and Burton, UK \\ ${ }^{3}$ Trauma \& Orthopaedics surgeon-Consultant, University hospitals of Derby and Burton, UK \\ ${ }^{4}$ Orthopaedic Surgeon, Department of Orthopaedics and musculoskeletal trauma, Louis \\ Pasteur university Hospital, Slovakia
}

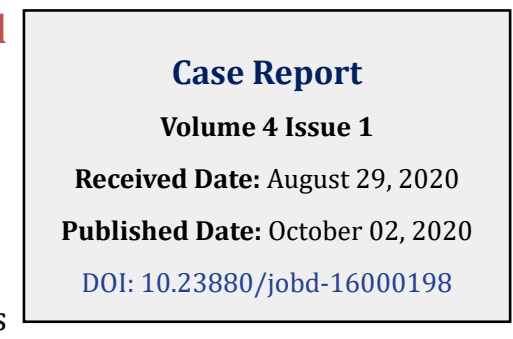

*Corresponding author: Ahmed Al Wadiya, Trauma \& Orthopaedics surgeon-Specialist, University hospitals of Derby and Burton. UK, Tel: +447903537880; Email: Ahmed.al-wadiya@nhs.net

\section{Abstract}

Sacrococcygeal pilonidal disease presentation varies greatly between being asymptomatic, acute abscess and chronic sinus. Acute abscess constitutes almost half of the initial presentation of such disease. To our best knowledge, acute abscess presentation hasn't been reported as a result of acute trauma in the literature. In this paper, we report a case of atypical presentation of sacrococcygeal pilonidal disease after a fall on the back a week earlier. Surgical management of the abscess, incidental finding of the pilonidal disease and lessons learned from such case is discussed in detail.

Keywords: Pilonidal Disease; Pilonidal Sinus; Sacrococcygeal; Presentation; Surgical Management

\section{Introduction}

Sacrococcygeal pilonidal disease is the cause for one of the most common surgical problems in young adults, where males are three times more affected than females. Pilonidal disease presentation ranges from an acute abscess to an asymptomatic pit along the inter-natal cleft, to a sluggish non-healing wound [1]. Risk factors include obesity, hirsutism and sedentary occupation, deep natal cleft and family history [1-3]. This case report describes a 23-year-old man with post-traumatic swelling at the lumbosacral region that was increasing in size over a week time and presented large abscess and systemically unwell. After laboratory investigation and radiography, a decision was made for surgical incision and drainage of that abscess. On drainage foul odour and content was addressed which doesn't correlate to such abscess. The general surgeons were called to investigate a communication to bowel, where a series of meticulous examination and rigid sigmoidoscope was done. A communicating pilonidal cyst was found and addressed surgically.

\section{Case Presentation}

A 23-year-old man presented to the emergency department with low back pain, bruising and a painful small lump in his lower back after a sustained fall week earlier landing on his back on a pavement whilst being out drinking. On examinations, the patient was generally well in himself, no fever was reported, extensive bruising over and swelling over the lumbosacral area which was not erythematous or warm on touch. He was able to fully bear weight without any aids. Laboratory works showed CRP of 44 and WCC of 14.9. A lumbosacral lateral radiograph showed normal lumbar lordotic curvature excluding any vertebral collapse or fracture. The clinical impression at this point was a hematoma; 
therefore the patient was reassured and sent home on oral antibiotics, analgesics and worsening advice. Advised to rest and apply ice to reduce the swelling. He presented 3 days later with increasing pain and swelling, and he reported fever over the previous night, on examination the patient was having feverish, with a temperature of 37.4-degree Celsius, systolic blood pressure of 105 , respiratory rate of 15 breath/ min, oxygen saturation of 99 on room air and pulse of 107

Local examination revealed that swelling has become tenderer and increased in size, at the centre skin started to break with the appearance of small yellow pustule. Urgent investigations were arranged including FBC, CRP, plain radiograph and CT scan of the lumbosacral spine. CRP was 269, and WBC was 19.7. The radiograph showed maintained alignment with some straightening of the normal lumbar lordotic curvature which was attributed to muscle spasm, both vertebral body heights and disc spaces were maintained with normal appearance of sacroiliac joints. The CT scan was requested due to the unavailability of Ultrasound and MRI scans out of hours. The Lumbosacral CT scan showed a large $(7.6 \times 6.2 \mathrm{~cm})$ collection posterior to the sacrum within the subcutaneous fat which was likely to represent a subacute haematoma and there was a solitary locule of gas within it raising the suspicion for an infected haematoma. The patient was started on intravenous broad-spectrum antibiotics and starved for incision and drainage (I\&D) in the theatre the next morning. The patient was operated on under general anaesthesia in floppy lateral posture. After prepping and draping, A 4-cm-incision was made the most fluctuant point and altered blood and pus came out from the sacral abscess, a sample taken for culture and sensitivity then finger introduced to open all loculi and foul smell and consistency matter was coming out, raising the suspicion of faecal substance and communication with bowel. The General surgery on-call team was immediately contacted, and they came within minutes. The General surgeons performed a digital rectal examination and a rigid sigmoidoscopy up to $23 \mathrm{~cm}$ which confirmed normal mucosa and absence of communication with the abscess. The surgical team reexplored the abscess site indicating that the abscess was superficial to the sacral fascia with no further faecal matter seen. There was an incidental finding of a pilonidal sinus communicating with the sacral abscess which was treated surgically in the same setting by off-midline incision and drainage. Three litres of Normal saline were used to washout the sinus and the sacral abscess and both were packed with Aquacel $囚$ dressing. As a result of the absence of abdominal symptoms, signs and previous CT findings, no abdominal exploration was indicated. The general surgeons took over patient care and he continued the I.V antibiotics for another 48 hours postoperatively. 48 hours later, after the wound was reviewed and showed healthy edges and clean dressing, antibiotics were stopped completely. He was discharged home after his blood works showed a significant drop of CRP to 150 and WBC to 16.4. The patient was followed up electively after that with regular dressing and packing allowing

The culture showed heavy growth of mixed anaerobes and coliform, moderate growth of Bacteroides and light growth of skin flora. Almost most samples were sensitive to metronidazole (Figure 1).

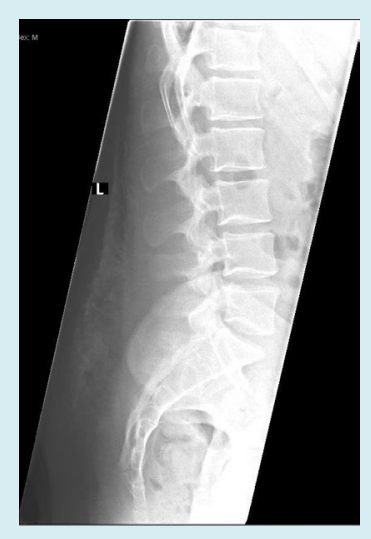

Figure 1: XR Lumbar Spine: Alignment is maintained with some straightening of the normal lumbar lordotic curvature which may be due to muscle spasm.

Vertebral body heights and disc spaces are maintained throughout, no vertebral collapse or fracture. Sacroiliac joints appear normal (Figure 2).

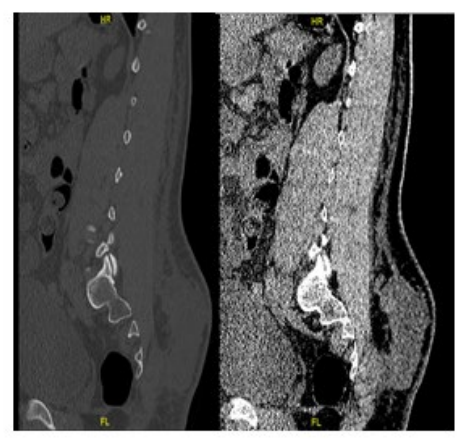

Figure 2: CT Lumbar Spine Findings: No evidence of a fracture or dislocation of the lumbar spine. Posterior to the sacrum within the subcutaneous fat, there is a large 7.6 x $6.2 \mathrm{~cm}$ collection which is likely to represent a subacute is haematoma and there is a solitary locule of gas within it and this raises the suspicion for an infected haematoma. No contrast has been given. No other significant finding. 


\section{Journal of Orthopedics \& Bone Disorders}

\section{Discussion}

Our patient shared the same characteristic risk factors for the sacrococcygeal pilonidal disease in terms of high body mass index, increased body hair, and deskbound occupation. The classical presentation of pilonidal disease varies significantly from asymptomatic pits to acute abscess and chronic sinus. Acute abscesses represent almost half of initial confronts for pilonidal disease. [1]. Regarding Acute pilonidal abscess presentation, Patients typically present with symptoms in the gluteal cleft in terms of fast onset of severe pain, tenderness, swelling, and redness which may be accompanied with drainage from the involved area. On examination, they usually have erythema and indurated mass, classically at the apex of the natal cleft. Oedema associated with soft tissue inflammation may obscure the characteristic appearance of pilonidal pits which may be visible in the midline of the gluteal cleft. These pits are the primary openings that may communicate with a subcutaneous epithelialized tract that connects with secondary openings. The secondary openings may be located off-midline, have granulation tissue surrounding the opening and/or drain purulent or serosanguinous material. Clinical examination alone is often sufficient to make the diagnosis, and except in rare cases of systemic illness or complex abscesses, imaging or laboratory workup is not necessary [4].

The points which differentiate our case from the classical picture are:

- Presence of fall history a week before presentation, which widens the differential diagnosis into trauma, fracture or osteomyelitis rather than pilonidal disease.

- The location of the abscess was far cephalic to the natal cleft about $8 \mathrm{~cm}$ which did not raise the suspicion of secondary opening for a sacrococcygeal pilonidal cyst.
Differential diagnosis:

1. Anorectal crytoglandular abscess extending into the natal cleft,

2. hidradenitis, and

3. low anorectal fistula such as seen in Crohn's disease

It is very difficult to predict the pathogenesis in such patient and whether he developed hematoma from such fall which then communicates with nearby the pilonidal disease and got infected or there was an already present pilonidal abscess which was exacerbated by the trauma.

Nevertheless, lessons learned from such case are:

$>$ A digital rectal examination with a rigid proctoscopy should be performed before operating upon a patient for pilonidal disease.

$>$ Meticulous examination of the patient, in terms of natal cleft examination, is always needed and abscess presentation even located laterally or far cephalic from natal cleft and history of recent trauma doesn't refute such examination.

\section{References}

1. Papaconstantinou HT, Thomas JS (2011) Pilonidal Disease and Hidradenitis Suppurativa. In: The ASCRS Textbook of Colon and Rectal Surgery, pp: 287-299.

2. Søndenaa K, Andersen E, Nesvik I, Søreide JA (1995) Patient characteristics and symptoms in chronic pilonidal sinus disease. Int J Colorectal Dis 10(1): 39-42.

3. Akinci ÖF, Bozer M, Uzunköy A, Düzgün ŞA, Coşkun A (1999) Incidence and aetiological factors in pilonidal sinus among Turkish soldiers. Eur J Surg 165(4): 339342.

4. Mattei P (2011) Fundamentals of pediatric surgery. Fundamentals of Pediatric Surgery. 